\title{
Design of one-dimensional photonic crystals using combination of band diagram and photonic gap map approaches
}

\author{
V. A. Tolmachev, ${ }^{1,2}$ T. S. Perova, ${ }^{1, a)}$ J. Ruttle, ${ }^{1}$ and E. V. Khokhlova ${ }^{1}$ \\ ${ }^{1}$ Department of Electronic and Electrical Engineering, University of Dublin, Trinity College, \\ Dublin 2, Ireland \\ ${ }^{2}$ Ioffe Physical-Technical Institute, Polytechnicheskaya 26, St. Petersburg 194021, Russia
}

(Received 16 May 2008; accepted 10 June 2008; published online 12 August 2008)

\begin{abstract}
The design of one-dimensional photonic crystals and the analysis of their optical properties have been performed using band diagram and forbidden gap map methods. It has been shown that the latter method is more useful for practical applications since (i) it can be applied for any number of periods and (ii) it is more suitable for the selection of a filling fraction in a wide range of values using a single graphical presentation. Three different types of photonic crystals with small, medium, and high optical contrast were modeled using both methods. The features of the omnidirectional band gap formation for photonic crystals with small optical contrast and low number of periods have been explained. The formation of a one-dimensional photonic structure with limited number of periods and omnidirectional band gap close to that for an infinite periodic structure has been discussed. (C) 2008 American Institute of Physics. [DOI: 10.1063/1.2963700]
\end{abstract}

\section{INTRODUCTION}

Microphotonics has been recently recognized as one of the ways to further the development of communication and information technologies. Its hardware component base is rapidly growing. It is recognized that the most important points for the development of new technologies are functionality, low cost, and accessibility. From these points of view, silicon is the most suitable material due to its low cost and well-developed complementary metal-oxide semiconductor processing. ${ }^{1}$ Silicon-based optoelectronics could provide a low-cost solution for high-speed interconnects for data centers and other enterprises. The enabling technologies rely on fundamental building blocks such as silicon-based laser, amplifier, and modulator. ${ }^{1,2}$ Recently new areas of research in microphotonics were opened up due to the elaboration of the theory and application of photonic crystals (PCs). PCs possess unique optical properties and can be used as reflectors, waveguides, attenuators of spontaneous atomic emission, devices for controlling emission in microcavities, and others. ${ }^{3-5}$ Furthermore, PCs have demonstrated their potential for application due to a number of microphotonic devices utilized recently. ${ }^{6}$ During the last decade particular attention has been paid to the development of devices based on onedimensional (1D) PCs, thanks to certain advantages of these devices over two- and three-dimensional (3D) PCs. These advantages are mainly related to their simpler fabrication, low cost, and the possibility of having simultaneous total reflection in a certain frequency (or wavelength) range for light at any incident angle $\varphi$ and polarization, i.e., to possess omnidirectional bands (ODBs). ${ }^{7-9}$

The design of a 1D PC normally starts with the selection of the wavelength $\lambda$ of its operation. Then, the materials with corresponding refractive indices, $n 1$ and $n 2$, for suitable pe-

\footnotetext{
a) Author to whom correspondence should be addressed: Tel.: +3531 8961432. FAX: +3531 6772442. Electronic mail: perovat@tcd.ie.
}

riodic structure fabrication are chosen. Based on the above selection, the geometric thickness of both components, $d 1$ and $d 2$, will be determined in accordance with the Bragg condition

$$
\lambda / 2=n 1 d 1+n 2 d 2
$$

where $n d$ is the optical thickness of the corresponding component.

For equal optical thicknesses of each component $n d$ $=\lambda / 4$, the maximum width of the stop band is achieved for the required wavelength $\lambda$. Several approaches can be used in order to determine the optical properties of a 1D PC. One of these is to calculate the band diagrams (BDs) (Ref. 10) for the selected filling fractions $f=d 1 /(d 1+d 2)$, where $d 1+d 2$ $=\Lambda$ is the lattice constant of the PC. The second approach based on the gap map (GM) presentation of photonic band gaps (PBGs) was described in Ref. 5. For drawing the GM, in principle, both the BD (Ref. 8) and the reflection spectra method ${ }^{11}$ can be used. The calculation of the reflection spectra of 1D PCs with a certain number of lattice periods, $m$, is normally performed using the transfer-matrix method (TMM). ${ }^{12}$ It was shown previously that the GM approach is very useful in the selection of ODB structures, ${ }^{8,13}$ as well as tunable ${ }^{11}$ and composite ${ }^{14}$ photonic structures since it allows quick and easy analysis of structures for a large set of spectra (normally around 100) to be done practically on one plot.

In this paper we apply the GM approach based on both the aforementioned methods for the design and analysis of optical properties of 1D PCs. First, we used BD calculations for periodic structures with an infinite number of periods and for one predicted filling fraction. By doing this we were able to determine the position and width of the PBGs including ODB. Following this, the GM method was applied, which enabled us to select the proper filling fraction in a large range of possible numbers of $m$ and incidence angles. The advantage of the GM approach is that the design of ODB from the 


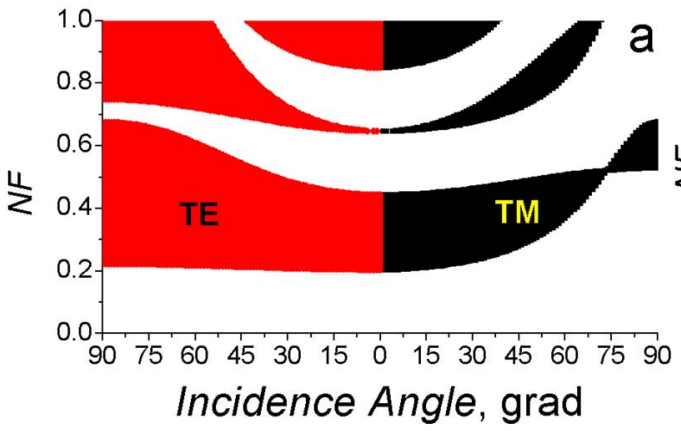

point of view of technical realization, including minimization of the number of periods $m$, is possible. Finally, in the selected wavelength range of ODB and using the obtained optimal filling fraction, the reflection spectra can be analyzed. With this final procedure we can obtain information about the features of the reflection spectra, namely, the maximum value of $R$, the spectra linewidth, the sharpness of the stop band edges, the presence of interference fringes between stop bands, and others. The approach suggested is demonstrated, by way of example, on the 1D PC, divided relatively into high, medium, and small refractive index contrast structures.

\section{BAND DIAGRAM METHOD}

To create BDs the Bloch solution was used in the same way as described in Ref. 10. Consider a 1D PC with an infinite number of layers. This means that a translational symmetry along the periodic direction will lead to a Bloch solution of the field component

$$
\begin{aligned}
\cos K \Lambda= & \cos \left(k_{1 x} d 1\right) \cos \left(k_{2 x} d 2\right) \\
& -M \sin \left(k_{1 x} d 1\right) \sin \left(k_{2 x} d 2\right),
\end{aligned}
$$

where $K$ is the Bloch wave vector and $k_{1 x}$ and $k_{2 x}$ are the $x$ components of the wave vectors $k_{1}$ and $k_{2}$ of the first and second constituent layers, respectively. The parameter $M$ is dependent on the polarization transverse-electric (TE) and transverse-magnetic (TM) accordingly,

$$
M \equiv\left\{\begin{array}{cl}
\frac{1}{2}\left(\frac{k_{2 x}}{k_{1 x}}+\frac{k_{1 x}}{k_{2 x}}\right), & \text { TE } \\
\frac{1}{2}\left(\frac{n_{1}^{2} k_{2 x}}{n_{2}^{2} k_{1 x}}+\frac{n_{2}^{2} k_{1 x}}{n_{1}^{2} k_{2 x}}\right) & \text { TM. }
\end{array}\right.
$$

The PBG exists in the range of frequencies (wave numbers) where the solution has a nonzero imaginary part. These frequencies are calculated and plotted versus the angle of incidence from the external medium onto the first layer. The external medium has a refractive index of 1 . This results in a graph similar to Fig. 1(x), where the normalized frequency $(\mathrm{NF})$ is $2 \pi c / \Lambda=\Lambda / \lambda$.

\section{THE GAP MAP APPROACH}

To create a GM, one must first calculate the reflection spectrum of the PC structure. This is achieved by calculating the optical response of PCs using TMM (Ref. 12). The 2 $\times 2$ complex matrix $S$ describes light propagation through the $\mathrm{PC}$

$$
S=\left[(I L)_{1}(I L)_{2}\right]^{m},
$$

where $I$ is the matrix that describes the interface between the two subscripted media, $L$ is the matrix that describes the subscripted media, and $m$ is the number of periods. $I$ and $L$ are based on the refractive index $n$ of the materials, the thickness $d$ of the materials, and the incident angle at which the light strikes the surface and its polarization.

Once the refractive index, incident angle, lattice constant, and the number of periods are chosen, the reflection spectrum of the structure is calculated using Eqs. (4) and (5) for a range of filling fractions from 0.01 to 0.99 ,

$$
R=\left|\left[S_{1,0} / S_{0,0}\right]^{2}\right| .
$$

For each of the reflection spectra, the region where the reflection was greater than 0.999 was recorded on the graph. Thus, the obtained PBG ranges were then plotted, together with all the other filling fractions, to give a graph called GM. The end result is displayed in Fig. 2(a) as an example. The graph gives a number of PBGs where the reflection is greater than 0.999 as a general rule, unless stated otherwise for some specific cases.

\section{PHOTONIC CRYSTALS WITH HIGH OPTICAL CONTRAST}

In this case we consider a photonic structure with a high optical contrast of $n 1 / n 2=3.42 / 1$ and optimum filling fraction for the second component at 0.774 from formula (1). The refractive index of the media for incoming and outgoing light beams is 1. For BDs shown in Fig. 1(a), the blueshift (to higher normalized frequencies) with increasing angle of incidence of PBGs for both polarizations is seen. The overlap of the lowest PBGs for the same condition is shown in Fig. 1(b). From the latter figure we can deduce that ODB in such PC cannot exist due to the fact that the short wavelength edge of the TM range for angles greater than $67^{\circ}$ does not have a mutual overlapping range with PBGs with normal incidence of light.

In order to investigate what the GM method reveals, GMs are calculated over a number of incident angles in the range of $0^{\circ}-85^{\circ}$ and for periods of 20 and 100 for both TE and TM polarizations (Fig. 2). From the GMs of TE polarization, as shown in Fig. 2, it can be seen that the regions of PBG are expanding with some blueshift to higher normalized 

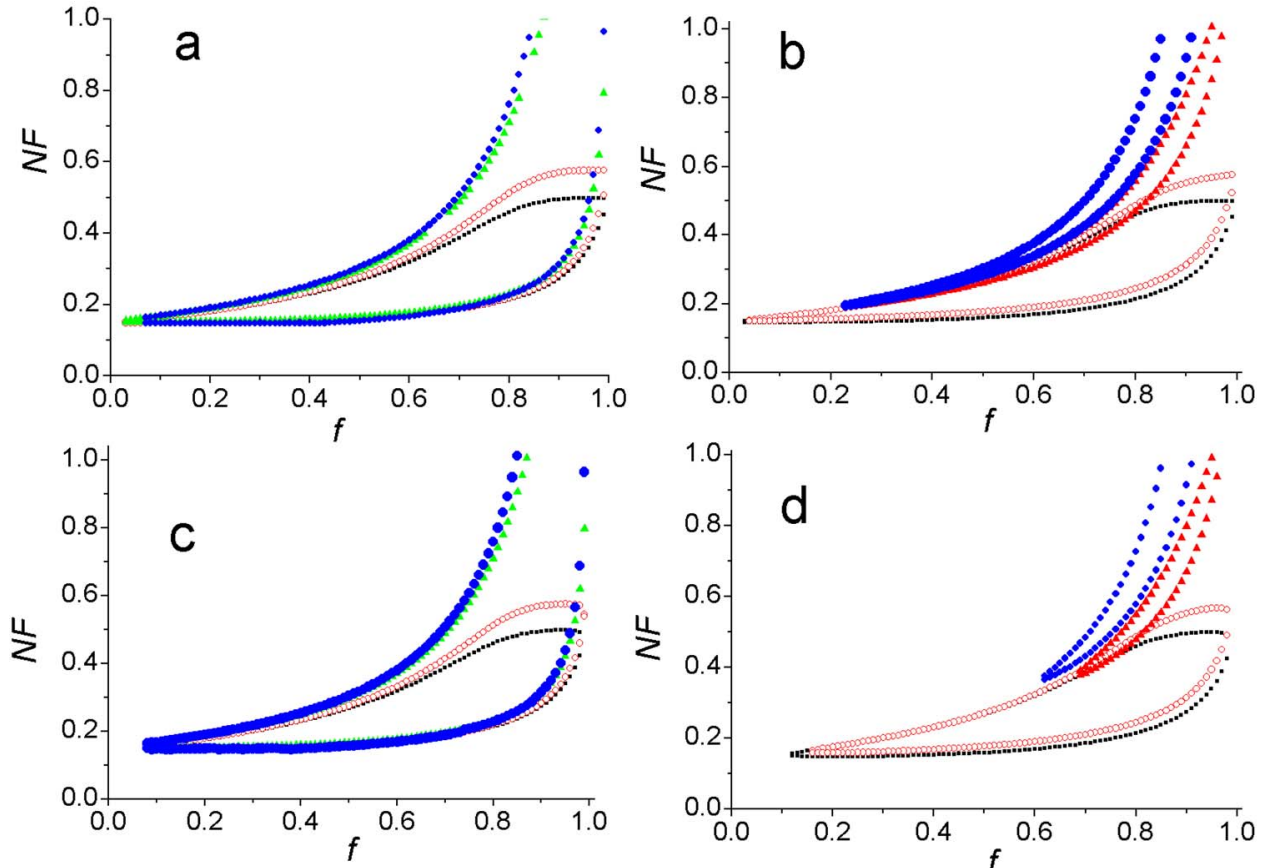
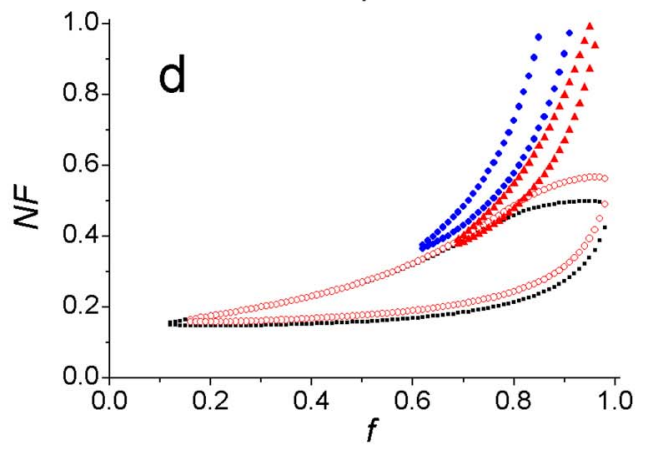

FIG. 2. (Color online) GMs showing the lowest PBG of a 1D PC with high optical contrast with a range of angle of incidences overlapped, number of period $m=100$ with (a) TE and (b) TM polarizations, $m=20$ with (c) TE and (d) TM. Incidence angle: $0^{\circ}$ (small solid square), $30^{\circ}$ (open circle), $67^{\circ}$ (solid triangle), and $85^{\circ}$ (solid circle). frequencies, as seen from the BD in Fig. 1. For the PBG regions of TM polarization the opposite is observed, namely, shrinkage of the PBG region with increasing angle of incidence. It can be seen in Figs. 2(b) and 2(d) that the PBG region with TM polarization and angle of incidence from $67^{\circ}$ to $85^{\circ}$ does not overlap with the PBG obtained for angle $0^{\circ}$. Therefore, for structures with high optical contrast, ODB cannot be obtained at any $f$ value.

\section{PHOTONIC CRYSTAL WITH MEDIUM OPTICAL CONTRAST}

As was already demonstrated in Fig. 1(b), ODB is defined by the range of overlapping of PBGs calculated for $0^{\circ}$ and maximal possible angles of incidence for TM and TE modes. The BDs for the photonic structure of medium optical contrast $n 1 / n 2=1.5 / 3.42$ with the optimum filling fraction of the first component at 0.695 are demonstrated in Fig. 3. In this case, the overlapping of the PBGs for TM and TE polarizations reveals an ODB. With a range of NF of $0.23-$ 0.3 this gives an ODB width $\Delta \mathrm{NF}$ of 0.07 . Since the TE is wider than the TM mode, the region of ODB is in fact forming from the width of the TM mode in the incident angle range from $0^{\circ}$ to $85^{\circ}$, as demonstrated in Figs. 1 and 3 . Therefore, Figs. 4(a) and 4(b) show only these two regions $\left(0^{\circ}\right.$ and $\left.85^{\circ}\right)$ for TM mode. The overlapping regions demonstrate large ODB areas for PC with $m=20$ and $m=10$.

To learn how the number of periods influences the formation of an ODB region, the corresponding dependences of $\Delta N F$ versus filling fraction $f$ were plotted using the data obtained from GM and BD calculations (see Fig. 5). From these graphs one can see that the parameters of the ideal PC (with infinite number of periods) are practically the same for PCs with 40 and more periods. Therefore, the PC structure possessing the ODB is totally formed at $m=40$. Although the PC with ODB can also be formed at $m<40$, the range of permissible $f$ values is narrowing along with the width of
ODB. It can also be seen that a quite substantial ODB region is observed for PC with six periods, and this is important from the point of view of $m$ minimization. In this case the range of permissible $f$ values with enough ODB is very small.

Figure 5 shows that a $\mathrm{PC}$ with $\Delta \mathrm{NF}=0.03-0.07$ can be formed from a photonic structure with a number of periods $m>20$ and $f$ varying in the range of 0.4 to 0.85 . Therefore, the design of medium contrast PC with omniband can be performed in a significantly wide range of spectra using a range of $\Delta \mathrm{NF}$, number of periods, and filling fractions.

\section{PC WITH SMALL OPTICAL CONTRAST}

The BDs of photonic structure with small optical contrast $n 1 / n 2=1.42 / 2.4$ and optimum filling fraction of the first component taken as 0.628 are demonstrated in Fig. 6. The overlapping of TM and TE PBGs shows that for both polarizations and any incident angle, there is a PBG in the range of $\mathrm{NF}=0.32-0.33$. Comparing this with a $\mathrm{PC}$ with medium contrast, the ODB region reduced down to $\Delta \mathrm{NF}$ $=0.01$. GMs for a number of incident angles with a range of

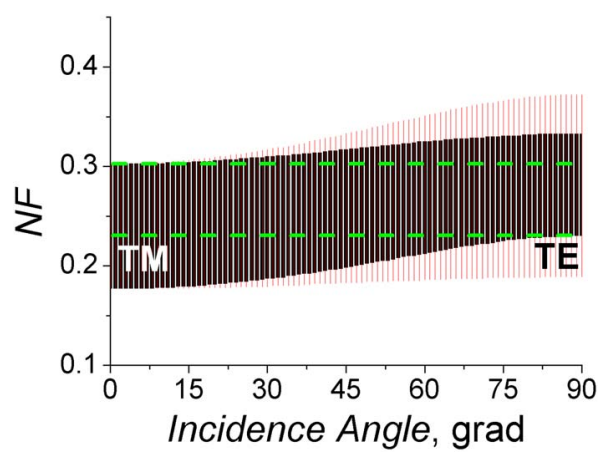

FIG. 3. (Color online) BD for TE and TM polarizations of a 1D PC with medium optical contrast $n 1 / n 2=1.5 / 3.42$ and the filling fraction of the first component $f_{1}=0.695$ overlapped with the ODB indicated by dashed lines. 

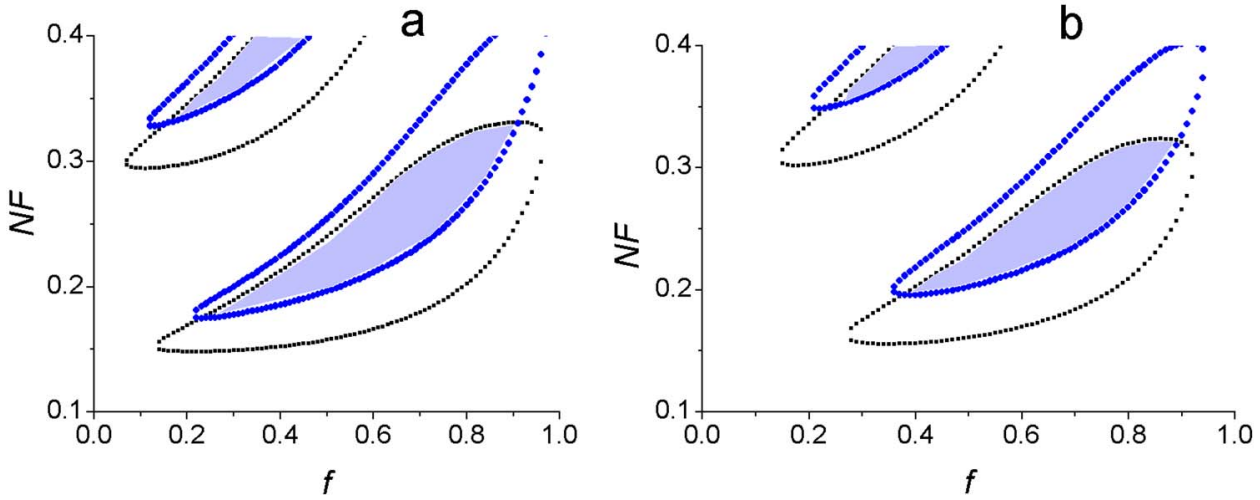

FIG. 4. (Color online) GM with the primary PBG region of a 1D PC with medium optical contrast for $0^{\circ}$ (small squares) and $85^{\circ}$ (circles) incident angles for TM polarization with the number of periods (a) 20 and (b) 10. Shaded regions correspond to ODB. $0^{\circ}-85^{\circ}$ and for a number of different lattice periods varying from 10 to 100 for TE and TM polarizations were calculated. Figure 7 shows GMs for $m=10$ and $m=20$. The same tendency for ODB shrinkage is seen when the number of periods decreases [Figs. 7(a)-7(c)]. It is worth noting that for PC with $m=10$, the PBG behaves slightly different with the TM PBG regions shrinking between $0^{\circ}$ and $44^{\circ}$, disappearing between $45^{\circ}$ and $82^{\circ}$, and reappearing and expanding from $83^{\circ}$ to $85^{\circ}$. This feature is presented in Fig. $7(\mathrm{~d})$ in more detail with an increased number of incident angles. These characteristics are not observed for the TE mode [see Fig. 7(c), $\mathrm{PBG}$ region for $60^{\circ}$.

In order to clarify this feature, the reflection spectra for TM polarization are demonstrated for $m=10$ and filling fraction $f_{1}=0.628$. These reflection spectra for $f=0.628$ are presented in Fig. 8(a) for different incident angles, including the range of angles where the $\mathrm{PBG}$ regions disappear. One can see that the PBG has a blueshift, as predicted, as the incident angle increases from $0^{\circ}$ to $85^{\circ}$. More details can be seen by looking at the reflection range from 0.99 to 1 . This shows that as the incident angle increases, the maximum reflection decreases below the selected criteria of 0.999 for the GM calculated in the range of angles from $45^{\circ}$ to $82^{\circ}$ [Fig. 8(b)]. It can also be seen from the reflection spectra shown in Fig. 8 that the width of the PBG is also dependent on the selection of the cutoff reflection value. For example, if a cutoff reflection value of 0.995 was chosen, then the PBG within a small range of normalized frequencies will appear in all angles of incidence on the GM plot.

The next step is to determine the ODB by using the GM method. In order to do this, GMs with different incident

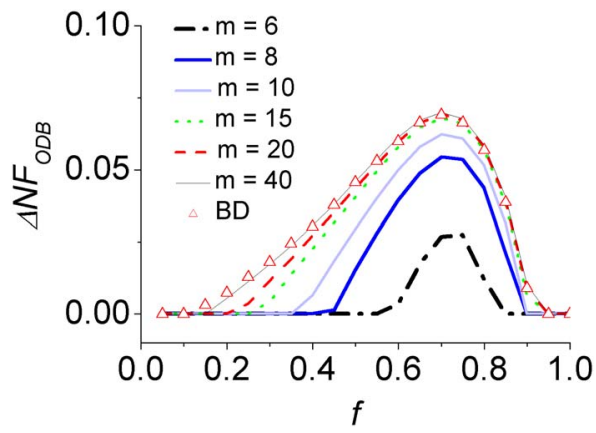

FIG. 5. (Color online) PC with medium optical contrast. Width of the ODB vs the filling fraction obtained for a number of periods using the GM method and for an infinite number of periods using the BD method. angles and polarizations must be overlapped. Since the PC with the number of periods $m=10$ is not good at reaching the reflection cutoff of 0.999 for certain incident angles (as mentioned above), we shall consider PCs with periods ranging from 15 to 100. It can be seen that an ODB for a PC with the number of periods $m=20$ can appear for a range of filling fractions from 0.44 to 0.7 [Fig. $7(\mathrm{~b})$ ]. The width $\Delta \mathrm{NF}$ can now be determined for a range of filling fractions and period numbers. These data were then placed on a graph in Fig. 9(a) to compare with the results obtained from the BD. The calculated curve for the PC with infinite number of periods is located lower than the one for the PC with $m=100$. This can be explained by the fact that the calculations for the first PC were carried out at $R=1$, while for the second PC we used the cutoff value of $R=0.999$. This resulted in the PBG for the second PC being slightly wider than the first PC. Similar to the previous case for PC with medium contrast, the analysis using the GM (or the set of reflection spectra for different $m$ values) gives the possibility to estimate the degree of ODB formation for a PC structure with properties close to the infinite $\mathrm{PC}$ in width $\Delta \mathrm{NF}=0.005-0.015$. As can be seen in Fig. 9(a), this is a PC with number of periods $m>40$ and $f$ $=0.4-0.7$. Thus, the formation of structures for the small contrast PC with ODB is starting at $m=15$ and completed at $m=40$.

It should be noted that the maximum position for the dependences $\Delta$ NF vs $f$, as shown in Figs. 5 and 9(a), does not correspond to the optimal $f$ value determined for the quota wavelength thickness and is shifted to the larger $f$

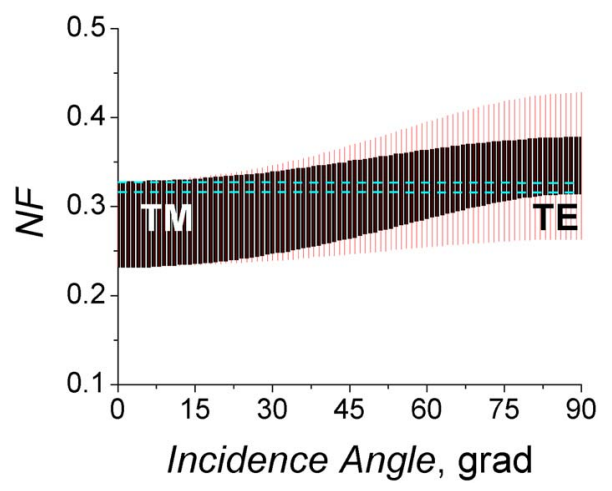

FIG. 6. (Color online) BD for TE and TM polarizations of a $1 \mathrm{D}$ PC with small optical contrast $1.42 / 2.4$ and $f=0.628$ with the ODB indicated by the two dashed lines. 

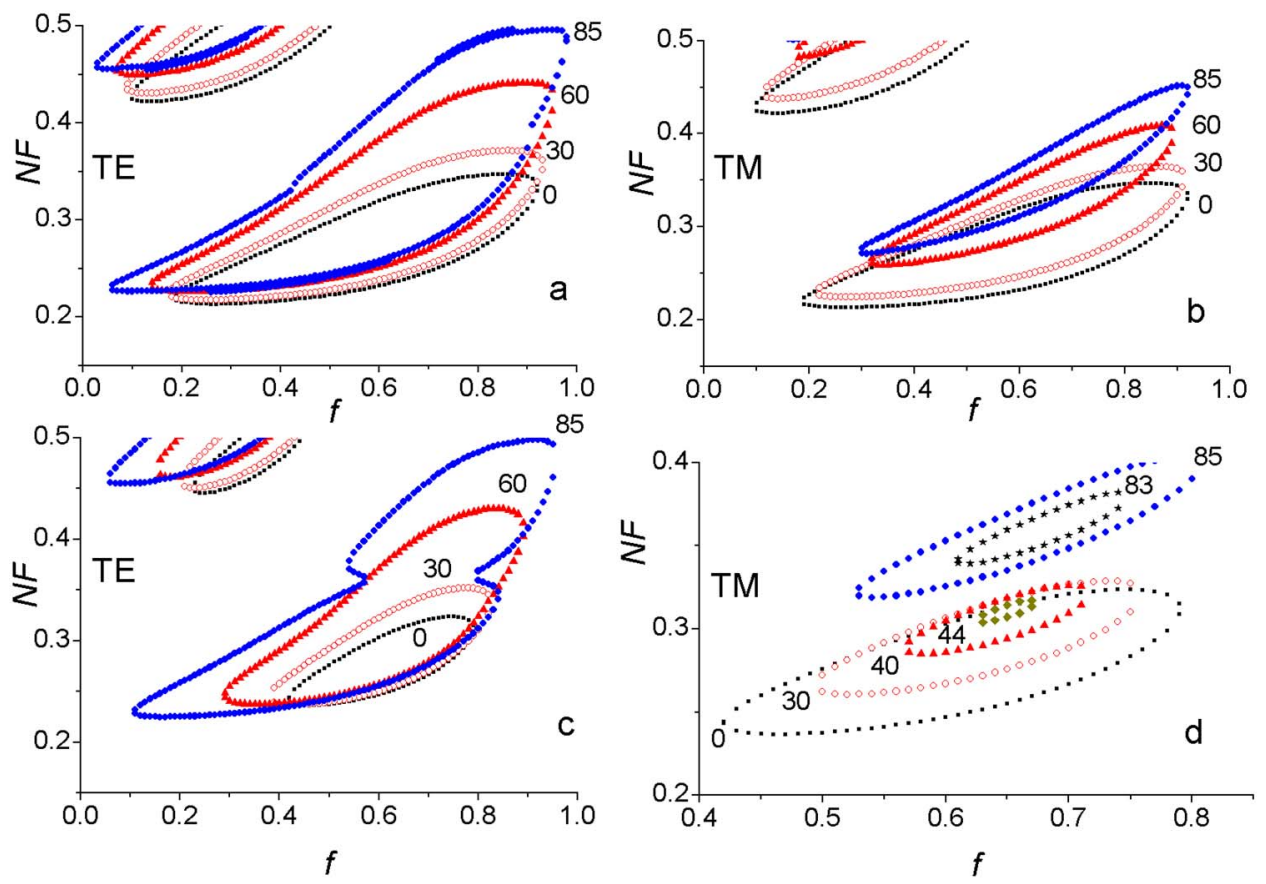

FIG. 7. (Color online) GMs for TM and TE polarizations showing the lowest PBG of a 1D PC with small optical contrast with a range of angles of incidences overlapped: (a) and (b) for $m=20$ and (c) and (d) for $m=10$.
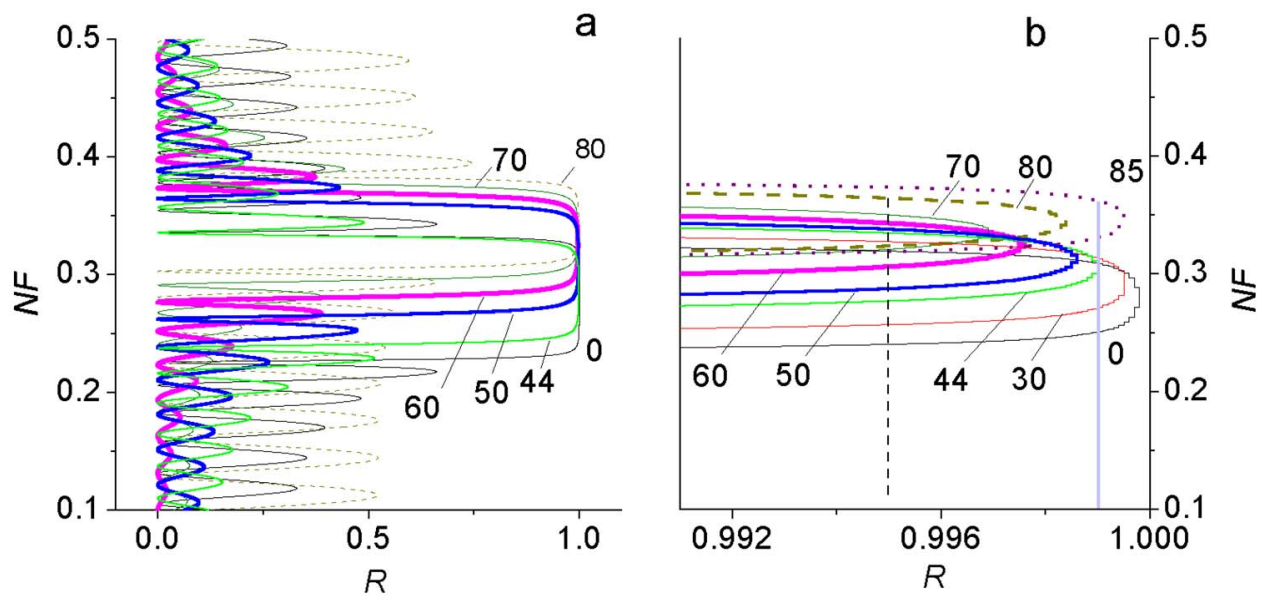

FIG. 8. (Color online) The TM reflection spectra of the PBG for 1D PC with small contrast calculated at different angles (indicated by numbers in grads) of incident light with the number of periods $m=10$ and filling fraction $f=0.628$. (a) Reflection spectra at different angles of incidence. (b) The spectra of PBG for the range of reflection from 0.99 to 1 . The vertical lines show the level (criteria) of reflection at 0.999 (gray line) and at 0.995 (black dashed line) for the selection of the PBG's edges used for the drawing of the PBG's regions on the GM.
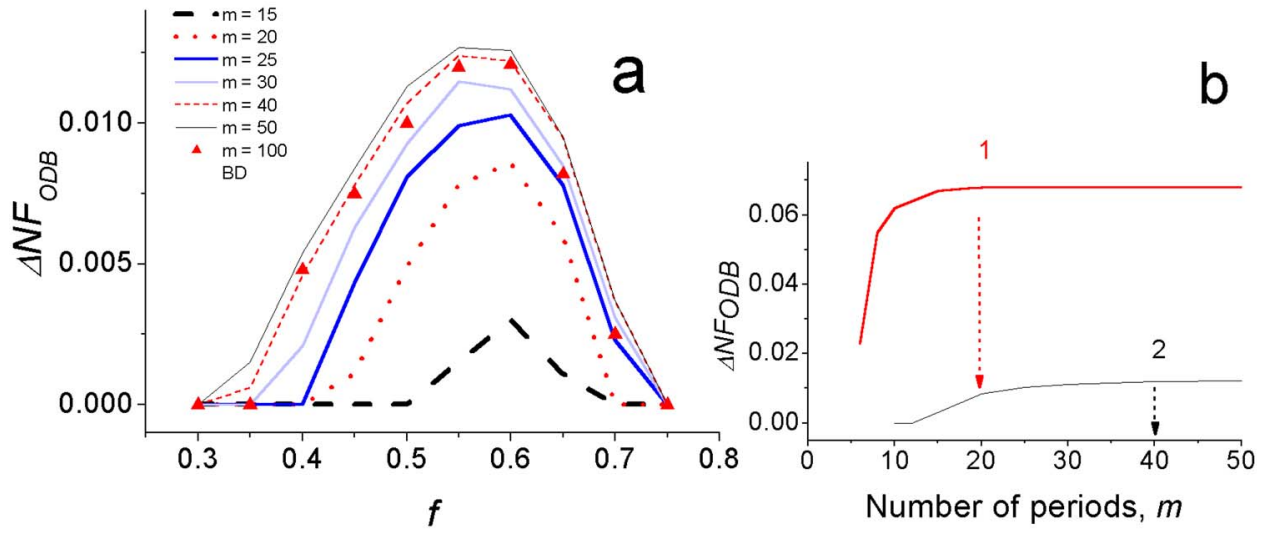

FIG. 9. (Color online) (a) Width of the ODB vs the filling fraction over a number of periods obtained using GM and BD methods for infinite number of periods for PC with small optical contrast. (b) Width of the ODB vs the number of periods for PC with medium optical contrast and $f=0.7$ (curve 1) and PC with small optical contrast and $f=0.6$ (curve 2). 
values for the low- and medium-contrast materials. This can be explained by the fact that in the first case (for quota wavelength layers), $f$ is determined for normal incidence of light, whereas in the second case it is determined by the difference in NF values for normal and oblique ( $85^{\circ}$ in our case) incidence of light. These features, as predicted in Ref. 8, must be taken into account during the design of PCs with ODB.

The intersection of this set of graphs [Fig. 9(a)] with line at $f=0.6$ demonstrates the dependence of $\Delta \mathrm{NF}$ on the number of lattice periods [Fig. 9(b), curve 2]. From this dependence it can be seen that as the number of periods increases, $\Delta \mathrm{NF}$ also increases, as well as the formation of the structure with $\triangle \mathrm{NF}$ parameters close to $\mathrm{PC}$, determined from $\mathrm{BD}$, and being completed for $m>40$. In a similar manner it can be concluded that there is complete formation of the mediumcontrast PC structure with ODB after $m=20$ (for $f=0.7$ ) [see Fig. 9(b), curve 1].

As mentioned above, the possibility of design and optimization of 1D PCs using GMs was demonstrated in Ref. 8. The distinction with our approach is that we are using the TMM for the calculation of the reflection spectra, based on which the PBG (and then GM) can be drawn. This enables us to design 1D PCs with finite number of lattice periods. Thus, using our approach in the course of optimization of the structure, another important parameter, namely, the number of lattice periods $m$, is included. Decreasing the number of periods can lead to a significant decrease in the PBG/ODB width, and therefore a reduction in the number of possibilities for the selection of the filling fraction.

Recently, another approach using reflection spectra calculations and their presentation on one 3D plot has been suggested in Ref. 15 for the design of PCs. From our point of view such presentation looks bulky and complicates the selection of the optimal PBG range. We believe that the GM presentation for optimization of ODB for 1D PC is more visual and clearer compared with the large set of spectra presented on a $3 \mathrm{D}$ plot.

\section{CONCLUSIONS}

In summary, it has been demonstrated that it is possible to model PCs using both the BD and the forbidden GM methods. From these methods we are able to discern whether an ODB is possible and what range of operation it would have. The advantage of the GM approach is that the design of ODB from the point of view of technical realization, including the minimization of the number of periods $m$, is possible. It was shown that ODB cannot be obtained at any possible filling fraction for high optical contrast photonic structures, as considered in this paper. PCs with ODB can be formed from photonic structures of medium optical contrast with a number of periods, starting from $m=6$ and $f$ varying in the range from 0.4 to 0.85 . A quite interesting behavior of PBGs has been observed on the GM for small contrast PCs with $m=10$. In particular, the disappearance of PBGs in TM polarization at certain angles of incidence was revealed. This effect was explained by the small decrease in the reflection coefficient in the PBG region. The formation of 1D photonic structures with an ODB gap close to an infinite periodic structure has been discussed.

\section{ACKNOWLEDGMENTS}

This work was supported by the Science Foundation Ireland (SFI) and Program RAS "Laser materials based on active media and optics of structured materials."

${ }^{1}$ A. Liu and M. Paniccia, Physica E (Amsterdam) 35, 223 (2006).

${ }^{2}$ R. Soref, IEEE J. Sel. Top. Quantum Electron. 12, 1678 (2006).

${ }^{3}$ E. Yablonovitch, Phys. Rev. Lett. 58, 2059 (1987).

${ }^{4}$ S. John, Phys. Rev. Lett. 58, 2486 (1987).

${ }^{5}$ J. D. Joannopoulos, R. D. Meade, and R. D. Winn, Photonic Crystals (Princeton University Press, Princeton, NJ, 1995).

${ }^{6}$ Photonic Crystals. Advances in Design, Fabrication, and Characterization, edited by K. Busch, S. Lölkes, R. Wehrspohn, and H. Föll (WileyVCH, Weinheim, 2004).

${ }^{7}$ Y. Fink, J. N. Winn, F. Shanhui, C. Chiping, J. Michel, J. D. Joannopoulos, and E. L. Thomas, Science 282, 1679 (1998).

${ }^{8}$ D. N. Chigrin, A. V. Lavrinenko, D. A. Yarotsky, and S. V. Gaponenko, Appl. Phys. A: Mater. Sci. Process. 68, 25 (1999).

${ }^{9}$ P. St. J. Russell, S. Tredwell, and P. J. Roberts, Opt. Commun. 160, 66 (1999).

${ }^{10} \mathrm{P}$. Yeh, Optical Waves in Layered Media (Wiley, New York, 1988).

${ }^{11}$ R. M. A. Azzam and N. M. Bashara, Ellipsometry and Polarized Light (North-Holland, Amsterdam, 1977).

${ }^{12}$ V. A. Tolmachev, T. S. Perova, and K. Berwick, Appl. Opt. 42, 5679 (2003).

${ }^{13}$ V. A. Tolmachev, T. S. Perova, and R. A. Moore, Opt. Express 13, 8433 (2005).

${ }^{14}$ V. A. Tolmachev, T. S. Perova, and K. Berwick, J. Appl. Phys. 99, 033507 (2006).

${ }^{15}$ H.-Y. Lee, H. Makino, T. Yao, and A. Tanaka, Appl. Phys. Lett. 81, 4502 (2002). 